\title{
Chemosaturation with Percutaneous Hepatic Perfusion: Outcome and Safety in Patients with Metastasized Uveal Melanoma
} Chemosaturation durch perkutane hepatische Perfusion
mit Melphalan bei hepatisch metastasiertem Aderhautmelanom:
eine Überlebens- und Sicherheitsanalyse

Authors

Cornelia Lieselotte Angelika Dewald ${ }^{1}$, Jan B. Hinrichs ${ }^{1}$, Lena Sophie Becker ${ }^{1}$, Sabine Maschke ${ }^{1}$, Timo C. Meine ${ }^{1}$, Anna Saborowski², Leon Jonas Schönfeld ${ }^{2}$, Arndt Vogel ${ }^{2}$, Martha M. Kirstein ${ }^{3}$, Frank K. Wacker ${ }^{1}$

\section{Affiliations}

1 Institute for Diagnostic and Interventional Radiology, Hannover Medical School, MHH, Hannover, Germany

2 Department of Gastroenterology, Hepatology and Endocrinology, Hannover Medical School, MHH, Hannover, Germany

$31^{\text {st }}$ Department of Medicine, University Medical Center Schleswig-Holstein Lübeck Campus, Lübeck, Germany

Key words

angiography, chemotherapy, interventional procedures, percutaneous, interventional oncology

received 21.07 .2020

accepted 04.01.2021

published online 03.02.2021

Bibliography

Fortschr Röntgenstr 2021; 193: 928-936

DOI 10.1055/a-1348-1932

ISSN 1438-9029

(c) 2021. Thieme. All rights reserved.

Georg Thieme Verlag KG, Rüdigerstraße 14,

70469 Stuttgart, Germany

Correspondence

Prof. Frank K Wacker

Institut für Diagnostische und Interventionelle Radiologie,

Medizinische Hochschule Hannover, Carl-Neuberg-Str. 1,

30625 Hannover, Germany

Tel.: $+49 / 511 / 5323421$

Fax: $+49 / 511 / 5329421$

wacker.frank@mh-hannover.de

\section{ABSTRACT}

Purpose Chemosaturation percutaneous hepatic perfusion (CSPHP) allows selective intrahepatic delivery of high dose cytotoxic melphalan in patients with curatively untreatable liver tumors while limiting systemic toxicity through hemofiltration of the hepatic venous blood. Aim of this study was to investigate the response to therapy, survival and safety of the CS-PHP procedure in patients with liver-dominant metastatic uveal melanoma (UM).
Materials and Methods Overall response rate (ORR) and disease control rate (DCR) were assessed according to Response Evaluation Criteria In Solid Tumors (RECIST1.1). Median overall survival (mOS), median progression-free survival (mPFS) and hepatic progression-free survival (mhPFS) were analyzed using Kaplan-Meier estimation. Adverse events were evaluated with Common Terminology Criteria for Adverse Events (CTCAE) v5.

Results Overall, 30 patients were treated with 70 CS-PHP in a salvage setting from October 2014 to January 2019. In total, ORR and DCR were $42.3 \%$ and $80.8 \%$, respectively. Overall, mOS was 12 (95\% confidence interval (Cl) 7-15) months, and both, mPFS and mhPFS were 6 months, respectively (95\% Cl 4-10; $95 \% \mathrm{Cl} 4-13$ ). Adverse events (AE) most frequently included significant but transient hematologic toxicities ( $87 \%$ of grade $3 / 4$ thrombocytopenia), less frequent AEs were hepatic injury extending to liver failure (3\%), cardiovascular events including one case of ischemic stroke (3\%). Conclusion Salvage treatment with CS-PHP is effective in selected patients with UM. The interventional procedure is safe. Serious hepatic and cardiovascular events, although rare, require careful patient selection and should be closely monitored.

Key Points:

- CS-PHP is safe for selected patients with liver-dominant metastatic uveal melanoma.

- CS-PHP resulted in hepatic disease control in $80 \%$ of patients.

- Hematologic events following CS-PHP are common but manageable.

\section{Citation Format}

- Dewald CL, Hinrichs JB, Becker LS et al. Chemosaturation with Percutaneous Hepatic Perfusion: Outcome and Safety in Patients with Metastasized Uveal Melanoma. Fortschr Röntgenstr 2021; 193: 928-936

\section{ZUSAMMENFASSUNG}

Ziel Die Chemosaturation mittels perkutaner hepatischer Perfusion mit Melphalan (CS-PHP) ist ein palliatives Therapie- 
verfahren für Patienten mit nicht kurativ behandelbaren Lebertumoren. Die CS-PHP erlaubt eine selektive intrahepatische Anreicherung von hochdosiertem Melphalan bei minimaler systemischer Toxizität durch venöse Hämofiltration. Ziel dieser Studie war es, das Ansprechen und Überleben sowie die Sicherheit der CS-PHP-Prozedur bei Patienten mit leberdominant metastasiertem Aderhautmelanom zu evaluieren.

Material und Methoden Gesamtansprechrate (overall response rate, ORR) und Krankheitskontrollrate (disease control rate, DCR) wurden anhand von Response Evaluation Criteria In Solid Tumors (RECIST1.1) ermittelt. Medianes Gesamtüberleben (mOS), medianes progressionsfreies Überleben (mPFS) und hepatisches mPFS (mhPFS) wurden mittels Kaplan-Meier-Schätzer ermittelt. Nebenwirkungen wurden entsprechend der einheitlichen Terminologie-Kriterien für Nebenwirkungen (CTCAE) v5 klassifiziert.
Ergebnisse 30 Patienten wurden zwischen Oktober 2014 und Januar 2019 mit 70 Chemosaturationen behandelt. Die ORR betrug 42,3\% und die DCR 80,8\%. Das mOS betrug 12 (95\%-Konfidenzintervall (KI) 7-15) Monate, das mPFS 6 (95\%-KI 4-10) und das mhPFS ebenfalls 6 (95\%-KI 4-13) Monate. Signifikante, aber transiente hämatotoxische Nebenwirkungen waren häufig (87\% Grad-3/4-Thrombozytopenie), hepatische Toxizität bis Leberversagen $(n=1 / 70)$ sowie kardiovaskuläre Komplikationen (ischämischer Insult, $n=1 / 70$ ) waren selten.

Schlussfolgerung Das palliative Therapiekonzept der Chemosaturation ist bei Patienten mit hepatisch metastasiertem Aderhautmelanom effektiv. Die interventionelle Prozedur ist sicher, seltene, aber schwerwiegende kardiovaskuläre und hepatische Komplikationen erfordern eine sorgfältige Patientenselektion und intensive Aufmerksamkeit.

\section{Introduction}

In the case of uveal melanoma (UM), the liver is often the first and solely affected organ. Patients with liver metastases have a dismal prognosis, with a median survival time of 4-15 months [1]. Although new findings in the field of targeted therapy and immunotherapy are affecting the prognosis of metastatic cutaneous melanoma [2, 3], there are no established systematic therapies available for metastatic UM [4]. Since the liver is the only organ affected by distant metastases in about $50 \%$ of cases [5], local hepatic therapies can result in delay of tumor progression and thus extend survival time with few side effects. Particularly with respect to inoperable or multifocal liver metastases, focus is developing on endovascular therapeutic approaches such as chemo-, radio- and immunoembolization and chemosaturation by percutaneous hepatic perfusion with melphalan (CS-PHP) [6].

CS-PHP is a minimally invasive therapeutic approach for the treatment of primary or secondary hepatic malignancies. Highdose melphalan is administered through a catheter in the hepatic artery and saturates the diseased liver tissue. Venous blood is aspirated through a double balloon catheter in the inferior vena cava (IVC), cleansed of melphalan using a specific extracorporeal filtration system, and returned to the systemic circulation transjugularly, minimizing systemic toxicity $[7,8]$.

The efficacy of CS-PHP was demonstrated in a Phase III study in patients with uveal and cutaneous melanoma. Compared with best available care (BAC), median progression-free survival (mPFS) and response rate improved after CS-PHP with the $1^{\text {st }}$ generation filter system [9]. A second-generation filter system with an improved filtration rate has been available since 2012. Since then, several studies have examined promising results regarding the safety and effectiveness of CS-PHP in patients with various primary and secondary tumors [3, 7, 8, 10-12]. Few studies have examined periinterventional safety and survival exclusively of patients with liver metastases due to $\operatorname{UM}[3,11]$; therefore there is a need for further, up-to-date data regarding the effectiveness of the 2nd generation CS-PHP technique (using the second-generation filter system).

The aim of this study was to provide an analysis of periinterventional complications, response, and survival of patients with hepatic metastatic uveal melanoma after palliative CS-PHP.

\section{Materials and Methods}

\section{Study Design/Patient Selection}

This retrospective single-center study was approved by the local ethics committee. CS-PHP was approved as an appropriate therapy in a multidisciplinary tumor conference. Prerequisites for CS-PHP were liver-dominant tumor involvement, sufficient hematologic, hepatic and renal function (hemoglobin $>8 \mathrm{~g} / \mathrm{dL}$; leukocytes $>2 \mathrm{tsd} / \mu \mathrm{L}$; platelets $>50 \mathrm{tsd} / \mu \mathrm{L}$; bilirubin $\leq 3 \times$ upper limit; serum creatinine $>60 \mu \mathrm{mol} / \mathrm{L}$ ). Patients were not treated if they had a history of heart failure (left ventricular ejection fraction $<40 \%$ ), significant chronic obstructive or restrictive airway disease, or a history of hemorrhage-prone intracranial lesions, apoplexy or transient ischemic attacks.

\section{Chemosaturation}

All CS-PHP procedures were performed under general anesthesia. An arterial catheter is positioned in the tumor-supplying hepatic arteries via a femoral sheath. A double-balloon catheter is inserted into the inferior vena cava and inflated to contain systemic spread of melphalan. The cranial balloon occludes the cavoatrial junction and the caudal balloon occludes the IVC below the hepatic veins ( $\triangleright$ Fig. 1). Venous blood can drain through the multiple lateral holes of the double balloon catheter. Once the correct position of the catheter is angiographically confirmed, a pump draws the venous melphalan-enriched blood into an extracorporeal filtration system, which separates up to $96 \%$ of melphalan from the blood $[13,14]$. The circuit is closed by returning the filtered blood via a CVC in the jugular vein. Then, melphalan 
$(2.5-3 \mathrm{mg} / \mathrm{kg}$ body weight, maximum $220 \mathrm{mg}$ ) dissolved in a 500 cc solution is infused through the arterial catheter in 100 cc portions. Intra-arterial nitroglycerin is applied in the event of flow-restricting vasospasm. An activated clotting time (ACT) greater than $500 \mathrm{~s}$ is required for safe extracorporeal filtration. For this purpose, $400 \mathrm{IE}$ heparin per $\mathrm{kg}$ body weight are initially applied IV. ACT is measured every 20-30 min during the intervention, and heparin is administered as needed. Following chemosaturation, venous blood is filtered extracorporeally for an additional $30 \mathrm{~min}$. Patients receive a single course of antibiotics and granulocyte colony-stimulating factor (G-CSF) 24-72 h after CS-PHP.

\section{Assessment of Response/Survival}

The first follow-up imaging (CT or MRI) was performed on average 7 weeks after CS-PHP. Response was assessed by Response Evaluation Criteria In Solid Tumors 1.1 (RECIST1.1) [15]. The overall response rate (ORR) was defined as partial response (PR) or complete remission (CR) and the disease control rate (DCR) as PR, CR or stable disease (SD) according to RECIST1.1. Median overall survival (mOS) was calculated after initial diagnosis of UM, after first diagnosis of hepatic metastasis, and after first CS-PHP until last follow-up or death (whichever occurred first). A comparison of the survival curves with respect to the line of therapy was performed. mPFS was calculated from the first intervention to progression (according to RECIST1.1); hepatic PFS (mhPFS) was calculated from first chemosaturation to hepatic progression, last follow-up, or death. Contraindications to further CS-PHP were progressive disease (PD) or poor tolerance of therapy.

\section{Assessment of Side Effects}

Periinterventional clinical reports, findings and laboratory values were retrospectively analyzed. Toxicity was classified according to Common Terminology Criteria for Adverse Events (CTCAEv5.0), which divided the severity of side effects into 5 grades (milddeath). Preintervention laboratory values were considered baseline, and laboratory values were recorded on days 1, 3, 7, 14 and 21 after CS-PHP. Major adverse cardiovascular events (MACE) and periinterventional mortality were analyzed.

\section{Statistical Analysis}

Data were obtained retrospectively from digital medical records. GraphPad Prism version 8.4.2 was used to determine response and survival with Kaplan-Meier estimators, comparisons were calculated using a logrank (Mantel-Cox) test. Continuous data were calculated using a Wilcoxon signed-rank test. A p-value of $<0.05$ was determined to be significant.

The study population is part of a current study analyzing the totality of chemosaturations performed at our institution for different primary tumor entities [16]. Part of the study population $(n=19)$ was already included in a single-center observational study [7].
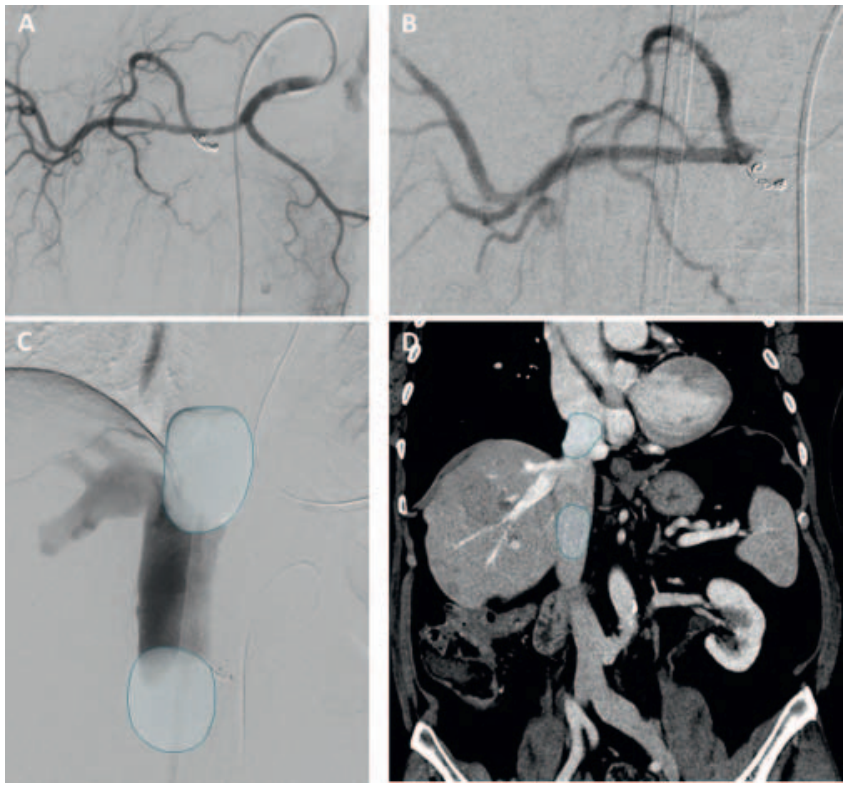

- Fig. 1 Chemosaturation procedure. A An arteriography of the coeliac truncus presents a characteristic arterial supply of the liver. The previously occluded right gastric artery does not show retrograde contrast flow. B The catheter is positioned in the proper hepatic artery proximal to the bifurcation into left and right hepatic artery (position of chemosaturation).After positioning of the arterial catheter in the tumor supplying artery, a double balloon catheter is placed and inflated in the inferior vena cava $\mathbf{D}$, the outlines of the double balloon are contoured in blue. D Schematic placement of the double balloon catheter in a coronary $\mathrm{CT}$ of the abdomen.

\section{Results}

\section{Patient and Intervention Data}

A total of 30 patients with hepatic metastatic UM were treated at our institution using CS-PHP between October 2014 and January 2019. The age of the patients was 57 (52-66) years (median and interquartile range). In 4 patients, initial diagnosis of UM showed synchronous hepatic metastasis; in the remaining patients, the median time between initial diagnosis of UM and first diagnosis of hepatic metastasis was 40 (17-73) months. The median time between initial diagnosis of hepatic metastasis and first CS-PHP was 5 (3-18) months. While $53 \%$ of patients received CS-PHP as first-line therapy for the treatment of hepatic metastases, the other patients were initially treated with other therapeutic approaches, receiving CS-PHP only as a second-to-fifth-line therapy ( $\triangleright$ Table 1 ).

A total of 5 (17.2\%) patients had extrahepatic tumor manifestations at the time of the first CS-PHP. Twenty-four patients showed a baseline LDH value of $>247 \mathrm{U} / \mathrm{I}$ (82.2\%). A total of 70 CS-PHP procedures were performed, with a maximum of 6 interventions per patient $(n=1 / 30)$. All CS-PHP procedures were performed with the second-generation filter system and were technically successful. - Table 1, 2 contain detailed demographic, clinical and interventional characteristics. 
- Table 1 Patient demographics and clinical parameters.

\begin{tabular}{|c|c|c|}
\hline parameter & value & percent \\
\hline \multicolumn{3}{|l|}{ gender } \\
\hline " male & 9 & 30 \\
\hline - female & 21 & 70 \\
\hline age (in years) ${ }^{* *}$ & $57(52-66)$ & \\
\hline \multicolumn{3}{|c|}{ LDH prior to $1^{\text {st }}$ CS-PHP* } \\
\hline . $\leq 247 \mathrm{U} / \mathrm{L}$ & 5 & 17.2 \\
\hline.$>247 \mathrm{U} / \mathrm{L}$ & 24 & 82.8 \\
\hline \multicolumn{3}{|c|}{ tumor volume prior to $1^{\text {st }} \mathrm{CS}-\mathrm{PHP}^{*}$} \\
\hline - $>30 \%$ & 8 & 27.6 \\
\hline . $\leq 30 \%$ & 21 & 72.4 \\
\hline \multicolumn{3}{|l|}{ extrahepatic tumor* } \\
\hline " no & 24 & 82.8 \\
\hline - yes & 5 & 17.2 \\
\hline \multicolumn{3}{|l|}{ ECOG score* } \\
\hline .0 & 19 & 65.5 \\
\hline . $1-2$ & 10 & 34.5 \\
\hline \multicolumn{3}{|c|}{ previous therapy of liver metastases } \\
\hline . SIRT & 4 & 13.3 \\
\hline - TACE & 2 & 6.7 \\
\hline - liver resection & 8 & 26.6 \\
\hline - chemotherapy & 5 & 16.7 \\
\hline - immunotherapy & 3 & 10 \\
\hline \multicolumn{3}{|c|}{ CS-PHP as treatment of liver metastases as } \\
\hline - $1^{\text {st }}$ line of therapy & 16 & 53.3 \\
\hline - $2^{\text {nd }}$ line of therapy & 7 & 23.3 \\
\hline - $3^{\text {rd }}$ line of therapy & 4 & 13.3 \\
\hline - $4^{\text {th }}$ line of therapy & 2 & 6.7 \\
\hline . $5^{\text {th }}$ line of therapy & 1 & 3.3 \\
\hline \multicolumn{3}{|c|}{$\begin{array}{l}\text { CS-PHP Chemosaturation Percutaneous Hepatic Perfusion; ECOG } \\
\text { Eastern Cooperative Oncology Group; RFA Radio Frequency Ablation; } \\
\text { SIRT Selective Internal Radiotherapy; TACE Transarterial Chemoembo- } \\
\text { lization. } \\
\text { "* Median with interquartile range. } \\
\text { " Data of 29/30 patients available. }\end{array}$} \\
\hline
\end{tabular}

\section{Response}

For response calculation, 26/30 (87\%) of patient data were available. After the first CS-PHP, 11 patients showed response (42.3\%), 10 patients SD (38.5\%), and 5 patients PD (19.3\%), including one patient with exclusively extrahepatic PD. The ORR was $42.3 \%$ $(n=11)$. Overall, disease stabilization was achieved in 21 patients (DSR: $80.8 \%$ ).
- Table 2 CS-PHP procedure characteristics.

\begin{tabular}{|c|c|c|}
\hline parameter & \multicolumn{2}{|l|}{ value } \\
\hline fluoroscopy time $(\min )^{*}$ & 10 & $(6-15.5)$ \\
\hline dose area product $\left(\mathrm{cGy} \times \mathrm{cm}^{2}\right)^{*}$ & 3926 & $(2539-7440)$ \\
\hline Melphalan dose $(\mathrm{mg})^{*}$ & 156 & $(145-177)$ \\
\hline lodine-based contrast $(\mathrm{ml})^{*}$ & 140 & $(110-160)$ \\
\hline intervention time (min)* & 178 & $(173-208)$ \\
\hline \multicolumn{3}{|c|}{ vasospasm treated with nitroglycerin } \\
\hline - after $1^{\text {st }}$ CS-PHP & \multicolumn{2}{|l|}{12} \\
\hline - after all CS-PHP treatments & \multicolumn{2}{|l|}{24} \\
\hline \multicolumn{3}{|l|}{ number of interventions/patients } \\
\hline - 1 & 13 & $(43.3 \%)$ \\
\hline .2 & 4 & $(13.3 \%)$ \\
\hline .3 & 6 & $(20 \%)$ \\
\hline . 4 & 5 & $(16.7 \%)$ \\
\hline - 5-6 & 2 & $(6.7 \%)$ \\
\hline $\begin{array}{l}\text { time between interventions } \\
\text { (days)* }\end{array}$ & 102 & $(66-141)$ \\
\hline $\begin{array}{l}\text { time from } 1^{\text {st }} \text { CS-PHP to } \\
1^{\text {st }} \text { follow-up imaging }\end{array}$ & 46 & $(39-70)$ \\
\hline $\begin{array}{l}\text { follow-up time after } \\
1^{\text {st }} \text { intervention (months) }\end{array}$ & 10 & $(2-15)$ \\
\hline \multicolumn{3}{|c|}{$\begin{array}{l}\text { CS-PHP chemosaturation percutaneous hepatic perfusion. } \\
{ }^{*} \text { Median with interquartile range. }\end{array}$} \\
\hline
\end{tabular}

\section{Survival}

After a median follow-up time of $10(2-15)$ months, the mOS after first CS-PHP at the time of data collection was 12 months (95\% Cl, 7-15 months). The mOS after initial diagnosis of liver metastases was 28 months ( $95 \% \mathrm{Cl} 17-32$ ). Comparison of survival curves after first diagnosis of liver metastases showed a trend toward prolonged median survival for patients receiving CS-PHP as first-line therapy (24.1 months) compared with those receiving second- and third-line therapy (23.6 months), although statistical significance was not achieved $(p=0.97)$. The mPFS after initial CSPHP was $6(95 \% \mathrm{Cl} 4-10)$ months, and mhPFS was also $6(95 \% \mathrm{Cl}$ 4-13) months ( $p=0.52)$. Fig. 2, 3 show the Kaplan-Meier curves of OS and PFS. At the time of the analysis 18 patients had died. Further CS-PHP procedures are planned for 10 of the 12 remaining patients ( $83 \%$ ). Individual survival and response times are shown in $>$ Fig. 4.

\section{Toxic Side Effects and further Complications}

After CS-PHP, a total of $87 \%$ of patients experienced clinically relevant thrombocytopenia (CTCAE Grade 3 or 4). Grade 3-4 anemia 
occurred in $40 \%$ and leukocytopenia in $10 \%$ of patients ( $>$ Table 3 ). On the whole, the myelosuppressive effect was transitory; blood levels regenerated within 3 weeks ( $\triangleright$ Fig. $\mathbf{5 a}-\mathbf{c}$ ). Platelet and red cell concentrates were administered in $23 \%$ and $17 \%$ of patients, respectively. Hepatic toxicity after CS-PHP regularly resulted in an increase (Grade 3-4) in liver enzymes (AST increase in 33\%; ALT increase in $23 \%$ of patients); less frequently, impairment of liver synthesis capacity was seen (hyperbilirubinemia in $10 \%$; hypoalbuminemia in $17 \%$ of patients), and a combined increase in ALT, AST, and bilirubin was seen in only one patient (3\%; Table 3 ).

The periinterventional mortality was $10 \%$; despite intensive medical measures, one patient died of sepsis 3 days after the first CS-PHP. Two additional patients died 3 and 12 days after the first intervention due to a combination of tumor lysis syndrome and

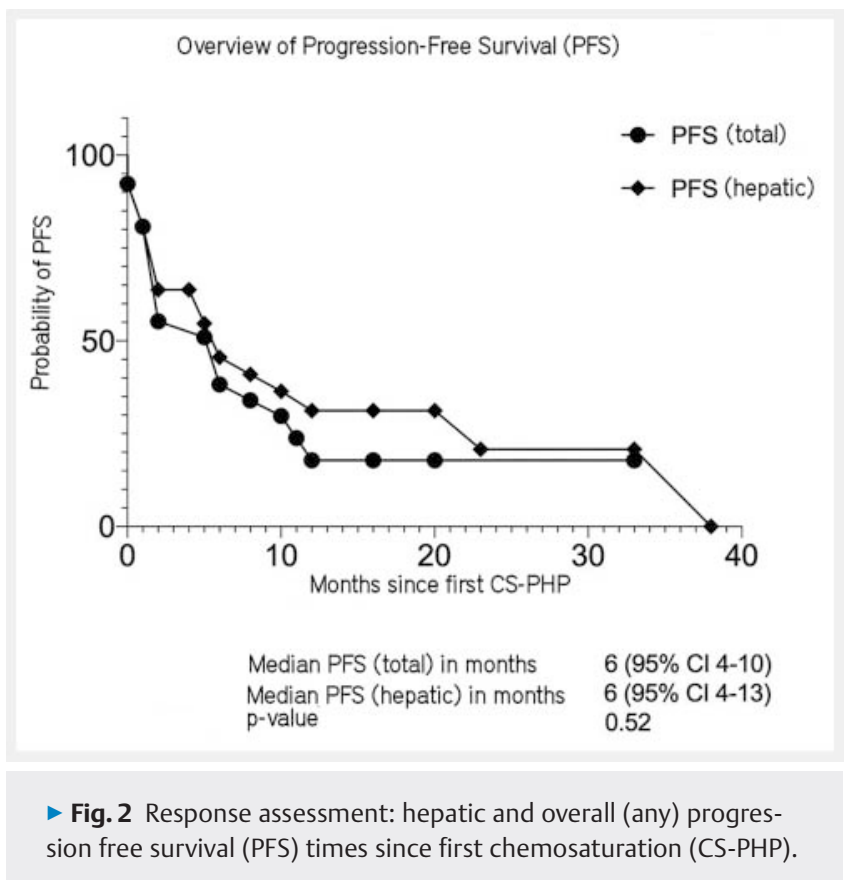

rapid tumor progression; even pre-interventionally, both patients had a high tumor burden with correspondingly high tumor volume (73\% and $32 \%$ ) and high LDH levels (3370 U/L and $3280 \mathrm{U} / \mathrm{L}$ ).

One patient suffered left cerebral artery occlusion the day after the first CS-PHP, whereupon immediate thrombectomy was performed (MACE rate $3 \%$ ). Due to persistent neurological symptoms, the patient was transferred to a neurological rehabilitation clinic.

Other non-hematotoxic and non-hepatotoxic postinterventional complications included iatrogenic hyperhydration and/or hypalbuminemia, which resulted in generalized or focal edema and ascites and/or pleural effusions after $11 \%$ of interventions and could be treated by diuretics and paracentesis. On the other hand, bleeding complications occurred after $10 \%$ of the procedures, in addition to noninterventional bleeding/hematoma at the puncture sites in one case each of ulcer bleeding (surgical care), epistaxis (tamponade), and ocular vascular hemorrhage (noninterventional).

\section{Conclusions}

The palliative therapy concept of CS-PHP is effective in patients with hepatic metastatic uveal melanoma. CTCAE Grade 3-4 hematologic and hepatic side effects are common but manageable. Cardiovascular events are rare but serious and must be considered in patient selection. Patients with high tumor burden often have serious complications and should be treated only in exceptional cases.

\section{Discussion}

Chemosaturation with melphalan is a novel technique ensuring high-dose chemotherapy of liver tumors with limited systemic toxicity. Patients with metastatic UM are particularly suitable candidates for this palliative therapeutic approach, as they often solely develop liver metastases which are sensitive to melphalan [17,

\section{a}

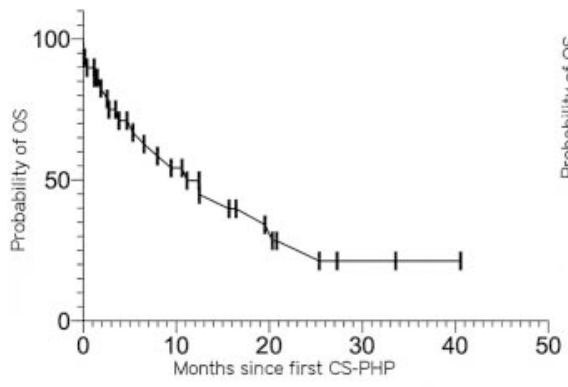

Median OS in months $\quad 12(95 \% \mathrm{Cl} 7-15)$

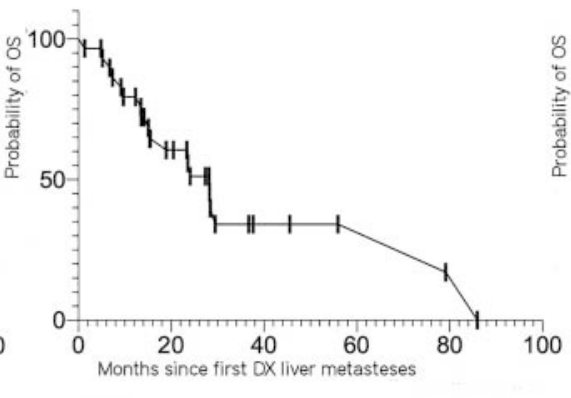

Median OS in months $\quad 28(95 \% \mathrm{Cl} 17-32)$

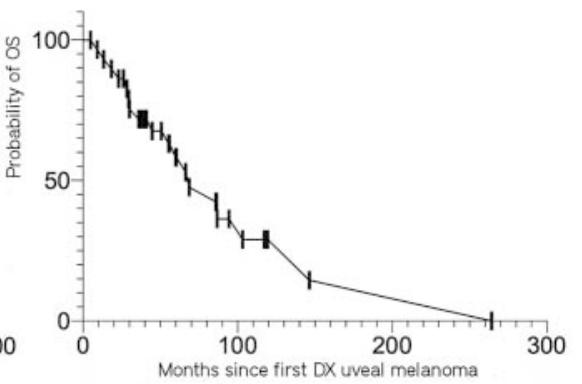

Median OS in months $69(95 \% \mathrm{Cl} 44-83)$

- Fig. 3 Survival assessment: overall survival times a after first chemosaturation (CS-PHP), $\mathbf{b}$ after first diagnosis of hepatic metastases and $\mathbf{c}$ after first diagnosis of uveal melanoma. 


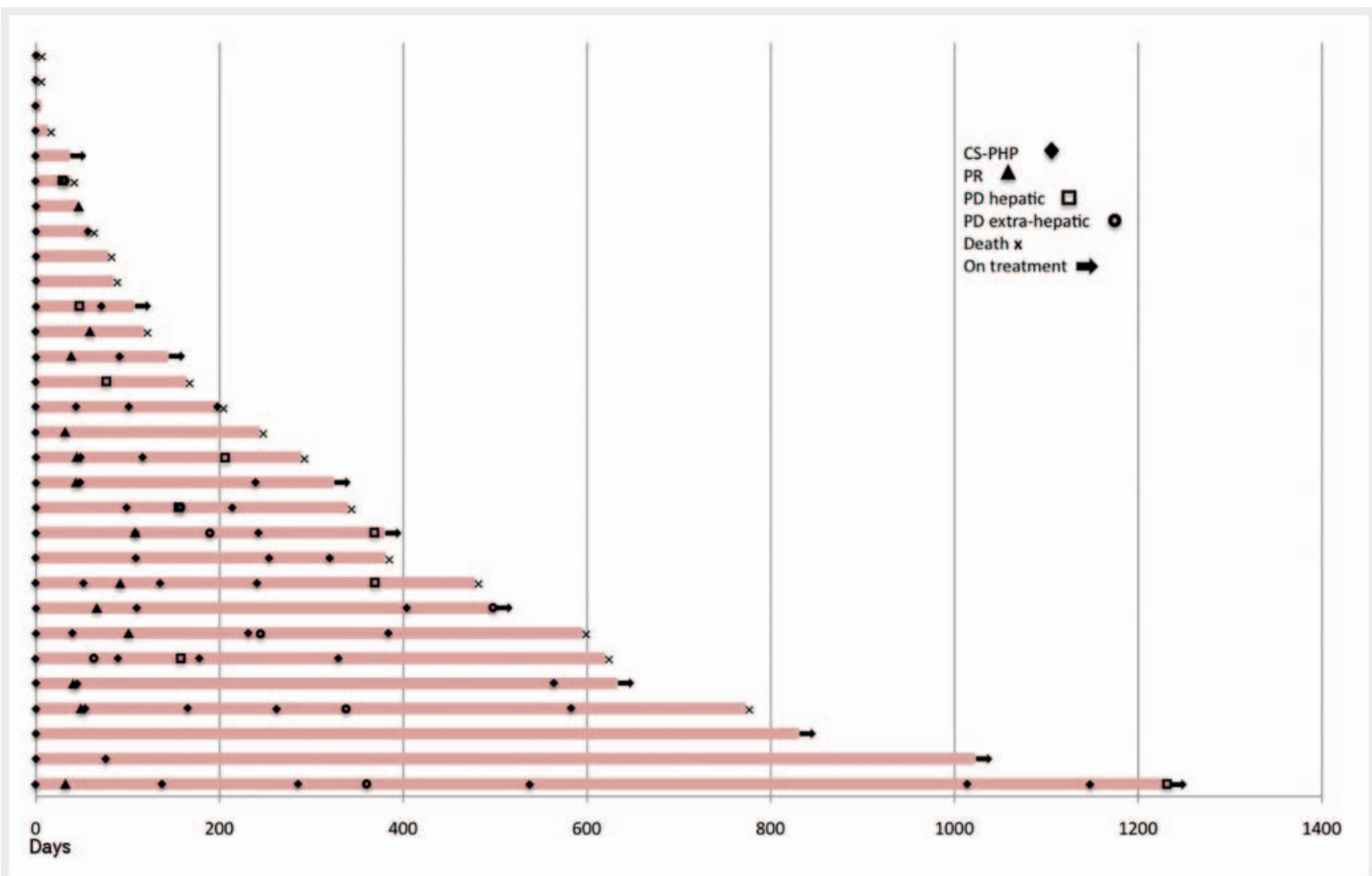

- Fig. 4 Individual survival times since first chemosaturation (CS-PHP) until last follow-up or death. Time to response, time to first hepatic and extra-hepatic progression and intervals between procedures are marked separately for each patient.

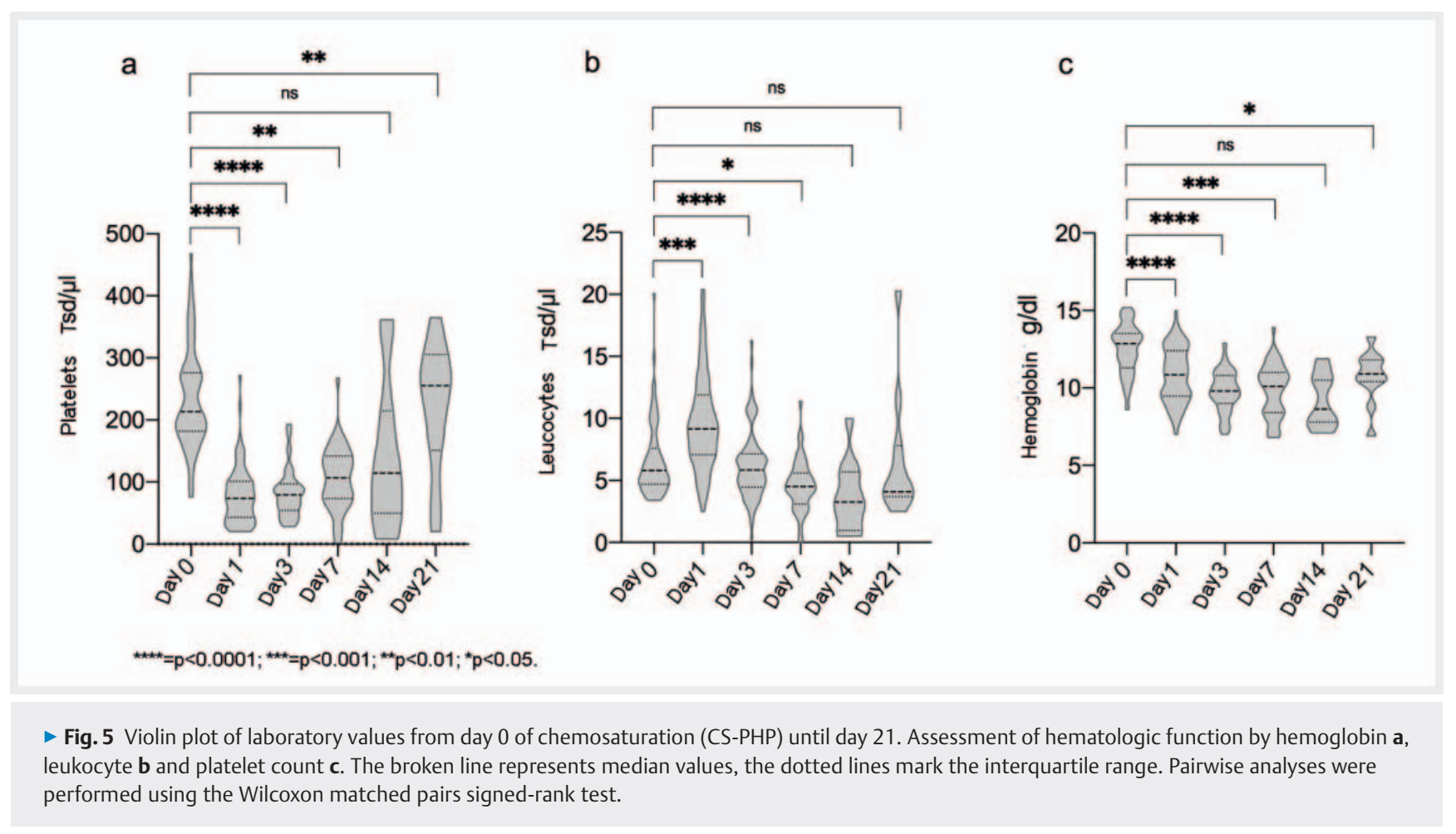


- Table 3 Hematological, hepatic and biliary adverse events grade 3 and 4 assessed by CTCAE v. 5 for each patient after first and all CS-PHP and assessed per CS-PHP procedure. Number (and percentage per patient) of transfused erythrocyte concentrates and platelet concentrates.

\begin{tabular}{|c|c|c|c|c|c|c|}
\hline & \multicolumn{2}{|c|}{$\begin{array}{l}\text { AE after first CS-PHP } \\
(n=30) / \text { per procedure }\end{array}$} & \multicolumn{2}{|c|}{$\begin{array}{l}\text { AE after all CS-PHPs/per } \\
\text { patient }(n=30)\end{array}$} & \multicolumn{2}{|c|}{$\begin{array}{l}\text { AE after all CS-PHPs/per } \\
\text { patient }(n=70)\end{array}$} \\
\hline & $\mathrm{n}=$ & $\%$ & $\mathbf{n}=$ & $\%$ & $\mathbf{n}=$ & $\%$ \\
\hline \multicolumn{7}{|l|}{ thrombenia } \\
\hline - grade 3 & 5 & 16.67 & 18 & 60 & 18 & 25.71 \\
\hline - grade 4 & 5 & 16.67 & 8 & 26.67 & 8 & 11.43 \\
\hline - grade $3+4$ & 10 & 33.33 & 26 & 86.67 & 26 & 37.15 \\
\hline \multicolumn{7}{|l|}{ leukopenia } \\
\hline - grade 3 & 0 & 0 & 1 & 3.34 & 1 & 1.43 \\
\hline - grade 4 & 2 & 6.67 & 2 & 6.67 & 2 & 2.86 \\
\hline - grade $3+4$ & 2 & 6.67 & 3 & 10.01 & 3 & 4.29 \\
\hline \multicolumn{7}{|l|}{ anemia } \\
\hline - grade 3 & 6 & 20 & 12 & 40 & 12 & 17.14 \\
\hline - grade 4 & 0 & 0 & 0 & 0 & 0 & 0 \\
\hline - grade $3+4$ & 6 & 20 & 12 & 40 & 12 & 17.14 \\
\hline \multicolumn{7}{|l|}{ AST increase } \\
\hline - grade 3 & 4 & 13.3 & 9 & 30 & 9 & 12.86 \\
\hline - grade 4 & 1 & 3.34 & 1 & 3.34 & 1 & 1.43 \\
\hline - grade $3+4$ & 5 & 16.67 & 10 & 33.34 & 10 & 14.29 \\
\hline \multicolumn{7}{|l|}{ ALT increase } \\
\hline - grade 3 & 2 & 6.67 & 7 & 23.34 & 7 & 10 \\
\hline - grade 4 & 0 & 0 & 0 & 0 & 0 & 0 \\
\hline - grade $3+4$ & 2 & 6.67 & 7 & 23.34 & 7 & 10 \\
\hline \multicolumn{7}{|l|}{ hyperbilirubinemia } \\
\hline - grade 3 & 1 & 3.34 & 3 & 10 & 3 & 4.29 \\
\hline - grade 4 & 0 & 0 & 0 & 0 & 0 & 0 \\
\hline - grade $3+4$ & 1 & 3.34 & 3 & 10 & 3 & 4.29 \\
\hline \multicolumn{7}{|l|}{ hypoalbuminemia } \\
\hline - grade 3 & 2 & 6.67 & 5 & 16.67 & 5 & 7.14 \\
\hline - grade 4 & 0 & 0 & 0 & 0 & 0 & 0 \\
\hline - grade $3+4$ & 2 & 6.67 & 5 & 16.67 & 5 & 7.14 \\
\hline platelet concentrate & 7 & 23.34 & 7 & 23.34 & & \\
\hline erythrocyte concentrate & 5 & 16.67 & 7 & 23.34 & & \\
\hline
\end{tabular}

18]. In addition, there is a lack of effective systemic therapies for metastatic UM [4], thus increasing the relevance of further development of minimally invasive approaches such as CS-PHP. The results of this single-center retrospective study demonstrate that CS-PHP is an effective method for treating patients with liverdominant metastatic UM.

After 70 CS-PHP procedures on 30 patients, there was an ORR of $42.3 \%$, mPFS and mhPFS of 6 months, and mOS of 12 months.
Our survival data are comparable to the landmark Phase III study for CS-PHP use, which identified improved tumor control (ORR $36 \%$, mPFS 5.4 and mhPFS 7 months) after CS-PHP of 93 patients compared to a study arm with BAC [9]. OS showed no significant difference between study arms in this study, which was influenced by a high crossover rate in the case of hepatic progression.

Regarding survival times in our study, it must be noted that CS-PHP was used relatively late after initial diagnosis of liver me- 
tastases. Furthermore, for various reasons, patients with far advanced tumor disease and a correspondingly poor prognosis were also treated. In principle, we strive to treat our patients promptly after diagnosis in order to improve the prognosis. This effort is often thwarted by delayed funding commitments from payers, who tend to be cautious despite a positive Phase III trial [9] and CS-PHP now recommended in guidelines [19]. Since only very few centers use the method, other local therapies are used due to the delay, which often leads to unnecessary tumor progression.

Due to a lack of randomized trials in metastatic UM, there is an absence of reliable data against which to measure the success of new therapies. The quality of most meta-analyses suffers from the limited comparability of the often small studies [20, 21]. Khoja et al. [22] analyzed 29 Phase II trials using the original data to establish a benchmark for mPFS and mOS, resulting in a significantly shorter mPFS (3.3 months) and mOS (10.2 months) compared to our data. In addition, response and survival data of locoregional liver therapies and various systemic therapies were compared, which showed superiority of locoregional approaches (mPFS 5.2; mOS 14.6 months) over systemic procedures (e. g., immunotherapy: mPFS 2.8 and mOS 8.9 months; chemotherapy: mPFS 2.6 and mOS 9.1 months). In order to further narrow down which patient group benefits most from which locoregional procedure, the implementation of a randomized study with different local therapies would be ideal; at the least, a registry study should be considered. As with any palliative procedure, quality of life and therapy costs should also be taken into account.

Even though systemic procedures and especially immunotherapy, which is successful in cutaneous melanoma, showed no benefit in Khoja et al. [22], a recent multicenter retrospective evaluation of combined checkpoint blockade in metastatic UM with mOS of 16.1 months provides a promising result, but with short mPFS (3 months) and low ORR (15.5\%) [23]. Ninety percent of the patients studied had liver metastases, $50 \%$ of whom had already been treated locally. In addition, patients had pulmonary metastasis in $36 \%$ of cases, osseous metastasis in $27 \%$, lymphatic metastasis in $19 \%$, and central nervous metastasis in $6 \%$. Only half of the patients had elevated pretherapeutic LDH - thus, hepatic tumor burden was not a primary concern, limiting comparability of patient cohorts and also therapies. Overall, CS-PHP should certainly not be viewed as a competitor to systemic therapy, but rather as an additive treatment modality for liver-dominant metastatic patients.

Concurring with several studies that investigated $2^{\text {nd }}$ generation CS-PHP in different patient cohorts (patients with exclusively metastatic UM $[3,10,11]$ or patients with various liver tumors [7, $8,12,16])$, hemotoxicity after CS-PHP in our study was significant, but treatable and transient. The literature reports the rate of transaminitis in patients with UM after CS-PHP to be between 6 and $20 \%$ [10]. In our study, an increase in AST or ALT was regularly observed, but insufficiency of liver function with Grade 34 transaminitis and hyperbilirubinemia was rare at $3 \%$.

A total of three deaths occurred postintervention. Two patients with high tumor burden died shortly after intervention from a combination of tumor progression and tumor lysis syndrome. In both patients, CS-PHP was performed despite the high risk because of explicit patient request. Another patient died of sepsis a few days after CS-PHP. Hughes et al. reported $6 \%$ deaths, including two patients with myelotoxicity-associated death and one patient with high tumor burden [9]. A negative correlation of high tumor volume and survival has been described previously [9-11], thus explicit interdisciplinary consideration of risk is essential in patients with high tumor burden.

Cardiovascular complications after CS-PHP are rare but can be severe. After intervention, one patient in this study suffered an ischemic cerebral infarction. Similar to our observations, isolated thromboembolic events after CS-PHP have been described in the literature [9-11]. Based on our experience and reports from other centers, an accurate history of cardiovascular risk factors is essential and contraindications should be taken into account.

A major limitation of this study is the retrospective study design. As a supraregional therapy center, we have treated a high number of patients; however, some were assigned to us over a long distance and in individual cases were monitored close to home; as a result complications could have been underestimated. Consideration of the side effects includes a number of influencing factors, such as anesthesia, balloon location, and intensive care follow-up, which, although specified in a protocol, are still subject to individual variation and may influence toxicity or other consequences.

In summary, our results show that for carefully selected patients with liver-dominant metastatic UM, CS-PHP is a safe palliative therapeutic approach that leads to effective hepatic tumor control beyond first-line therapy. Therapy should be started as early as possible, since advanced tumor disease leads to poorer outcomes and side effects with few available treatment alternatives.

\section{RELEVANCE OF THE STUDY}

- There is a lack of established systemic therapies for metastatic uveal melanoma, bringing local tumor therapy and CS-PHP in particular into focus.

- For carefully selected patients with liver-dominant metastatic UM, CS-PHP is a safe palliative therapeutic approach that leads to effective hepatic tumor control with limited systemic side effects.

\section{Conflict of Interest}

The authors declare that they have no conflict of interest.

\section{References}

[1] Yang J, Manson DK, Marr BP et al. Treatment of uveal melanoma: where are we now? Ther Adv Med Oncol 2018; 10: 175883401875717

[2] Larkin J, Chiarion-Sileni V, Gonzalez R et al. Combined Nivolumab and Ipilimumab or Monotherapy in Untreated Melanoma. N Engl J Med 2015; 373: 23-34 
[3] Karydis I, Gangi A, Wheater MJ et al. Percutaneous hepatic perfusion with melphalan in uveal melanoma: A safe and effective treatment modality in an orphan disease. J Surg Oncol 2018; 117: 1170-1178

[4] Triozzi PL, Singh AD. Adjuvant Therapy of Uveal Melanoma: Current Status. Ocul Oncol Pathol 2015; 1 (1): 54-62

[5] Development of Metastatic Disease After Enrollment in the COMS Trials for Treatment of Choroidal Melanoma: Collaborative Ocular Melanoma Study Group Report No. 26. Arch Ophthalmol 2005; 123: 1639

[6] Abbott AM, Doepker MP, Kim Y et al. Hepatic Progression-free and Overall Survival After Regional Therapy to the Liver for Metastatic Melanoma. American Journal of Clinical Oncology 2018; 41: 747-753

[7] Kirstein MM, Marquardt S, Jedicke N et al. Safety and efficacy of chemosaturation in patients with primary and secondary liver tumors. J Cancer Res Clin Oncol 2017; 143: 2113-2121

[8] Marquardt S, Kirstein MM, Brüning R et al. Percutaneous hepatic perfusion (chemosaturation) with melphalan in patients with intrahepatic cholangiocarcinoma: European multicentre study on safety, short-term effects and survival. Eur Radiol 2019; 29: 1882-1892

[9] Hughes MS, Zager J, Faries M et al. Results of a Randomized Controlled Multicenter Phase III Trial of Percutaneous Hepatic Perfusion Compared with Best Available Care for Patients with Melanoma Liver Metastases. Ann Surg Oncol 2016; 23: 1309-1319

[10] Meijer TS, Burgmans MC, Fiocco M et al. Safety of Percutaneous Hepatic Perfusion with Melphalan in Patients with Unresectable Liver Metastases from Ocular Melanoma Using the Delcath Systems' Second-Generation Hemofiltration System: A Prospective Non-randomized Phase II Trial. Cardiovasc Intervent Radiol 2019; 42: 841-852

[11] Artzner C, Mossakowski O, Hefferman G et al. Chemosaturation with percutaneous hepatic perfusion of melphalan for liver-dominant metastatic uveal melanoma: a single center experience. Cancer Imaging 2019; 19: 31

[12] Vogl T, Zangos S, Scholtz ] et al. Chemosaturation with Percutaneous Hepatic Perfusions of Melphalan for Hepatic Metastases: Experience from Two European Centers. Fortschr Röntgenstr 2014; 186: 937-944
[13] Vogel A, Gupta S, Zeile M et al. Chemosaturation Percutaneous Hepatic Perfusion: A Systematic Review. Adv Ther 2016; 33: 2122-2138

[14] Moeslein FM, McAndrew EG, Appling WM et al. Evaluation of Delcath Systems' Generation 2 (GEN 2) Melphalan Hemofiltration System in a Porcine Model of Percutaneous Hepatic Perfusion. Cardiovasc Intervent Radiol 2014; 37: 763-769

[15] Schwartz LH, Litière S, de Vries E et al. RECIST 1.1-Update and clarification: From the RECIST committee. European Journal of Cancer 2016; 62: $132-137$

[16] Schönfeld L, Hinrichs JB, Marquardt S et al. Chemosaturation with percutaneous hepatic perfusion is effective in patients with ocular melanoma and cholangiocarcinoma. J Cancer Res Clin Oncol [Internet] 20. Juni 2020 [zitiert 8. Juli 2020]; Verfügbar unter: http://link.springer.com/ 10.1007/s00432-020-03289-5

[17] Feldman ED, Pingpank JF, Alexander HR. Regional Treatment Options for Patients With Ocular Melanoma Metastatic to the Liver. Ann Surg Oncol 2004; 11: 290-297

[18] Jovanovic P, Mihajlovic M, Djordjevic-Jocic J et al. Ocular melanoma: an overview of the current status. Int J Clin Exp Pathol 2013; 6: 1230-1244

[19] S3-Leitlinie zur Diagnostik, Therapie und Nachsorge des Melanoms, Version 3.2, AWMF, Register-Nummer: 032/024OL 2019.

[20] Rantala ES, Hernberg M, Kivelä TT. Overall survival after treatment for metastatic uveal melanoma: a systematic review and meta-analysis. Melanoma Research 2019; 29: 561-568

[21] Rowcroft A, Loveday BPT, Thomson BNJ et al. Systematic review of liver directed therapy for uveal melanoma hepatic metastases. HPB 2020; 22 : 497-505

[22] Khoja L, Atenafu EG, Suciu S et al. Meta-analysis in metastatic uveal melanoma to determine progression free and overall survival benchmarks: an international rare cancers initiative (IRCI) ocular melanoma study. Annals of Oncology 2019; 30: 1370-1380

[23] Heppt MV, Amaral T, Kähler KC et al. Combined immune checkpoint blockade for metastatic uveal melanoma: a retrospective, multi-center study. j immunotherapy cancer 2019; 7: 299 